\title{
INTERVENÇÃO FÍSICA PARA TRATAR FRAGILIDADE EM IDOSOS COMUNITÁRIOS: REVISÃO SISTEMÁTICA
}

\section{Luciana Caetano Fernandes*1, Guilherme Augusto Santos Bueno¹, Lorraine Barbosa Cordeiro ${ }^{2}$ and Ruth Losada de Menezes ${ }^{3}$}

${ }^{1}$ Aluno(a) no Programa de Pós-Graduação em Ciências e Tecnologias em Saúde, Universidade de Brasília - UnB, Brasília-Brasil; ${ }^{2}$ Aluna do curso de Fisioterapia - Universidade Evangélica de Goiás - Unievangélica, Anápolis Brasil; ${ }^{3}$ Orientadora no Programa de Pós-Graduação em Ciências e Tecnologias em Saúde, Universidade de Brasília - UnB, Brasília-Brasil

\section{ARTICLE INFO}

\section{Article History:}

Received $20^{\text {th }}$ March, 2021

Received in revised form

$11^{\text {th }}$ April, 2021

Accepted $06^{\text {th }}$ May, 2021

Published online $26^{\text {th }}$ June, 2021

Key Words:

Aged. Frailty,

Exercise, Biomarkers.

*Corresponding author:

Luciana Caetano Fernandes

\begin{abstract}
Objective: Check if the physical intervention reverses the fragility, also evaluating biomarkers associated with this condition. Method: Following the Preferential Report for Systematic Reviews and Meta-analyzes (PRISMA statement), a search for articles of physical intervention was carried out in the last ten years, in the database "National Library of Medicine" (PubMed) database; "Scientific Electronic Library Online" (Scielo), "Physiotherapy Evidence Database" (PEDro) using the descriptors: Older adults AND Physical activity AND Frailty. Results: Two hundred and forty-one articles were found, sixty-two of which were eligible for reading. In this review, eight studies were carried out. All selected articles scored five or more points on the PEDro scale. Only one intervention study did not observe a reversal of frailty. As for the existence of a biomarker, it was not detected in these studies, although inflammation is a key point in the frailty syndrome. Conclusion: Through this study it was possible to show that combined interventions (physical intervention associated with another type of intervention) and physical interventions have a potential to reverse frailty in the elderly, with the combined intervention being better, which involves resistance training.
\end{abstract}

Copyright (C) 2021, Luciana Caetano Fernandes et al. This is an open access article distributed under the Creative Commons Attribution License, which permits unrestricted use, distribution, and reproduction in any medium, provided the original work is properly cited.

Citation: Luciana Caetano Fernandes, Guilherme Augusto Santos Bueno, Lorraine Barbosa Cordeiro and Ruth Losada de Menezes. "Intervenção física para tratar fragilidade em idosos comunitários: revisão sistemática. ”, International Journal of Development Research, 11, (06), $47939-47946$.

\section{INTRODUCTION}

O envelhecimento é um processo heterogêneo entre os seres humanos. A maioria das pessoas desenvolvem doenças e incapacidades ao envelhecer, porém a trajetória e a velocidade de mudanças no estado de saúde e da funcionalidade variam amplamente entre os indivíduos, independentemente da idade cronológica. Diferentes fatores como presença de comorbidades, subnutrição e obesidade, podem afetar a idade biológica, prejudicando o estado de saúde e funcionalidade, contribuindo para o surgimento de fenótipos como a fragilidade (Varadhan et al., 2008). A fragilidade é uma síndrome biológica caracterizada pela redução da capacidade de reserva em diferentes sistemas fisiológicos ao longo da vida que tornam o indivíduo mais vulnerável a agentes estressores. Essa síndrome se associa a variados desfechos desfavoráveis tais como: quedas, hospitalização, institucionalização e morte (Fried et al., 2001;
Fried et al., 2004). O estudo de Collard et al., 2012 com dados de 21 estudos de coorte, observou prevalência de fragilidade que variou de $4,0 \%$ a $59,1 \%$. essa grande variação pode ser explicada pelo uso de diferentes definições operacionais de fragilidade. existem mais de 20 tipos de instrumentos para avaliar a fragilidade (Dent; kowal; hoogendijk, 2016), sendo o mais utilizado o instrumento proposto por fried (fried et al., 2001; fried et al., 2004), onde existem mais de 200 tipos de adaptações no instrumento proposto por fried (Theou et al., 2015). a síndrome da fragilidade está associada a alterações imunológicas, endócrinas, com a perda de massa muscular (Fried et al., 2001; Fried et al., 2004) e o inflammaging é considerado importante contribuinte ao processo fisiopatológico da síndrome (Chen; Mao; Leng, 2014), contribuindo para a sarcopenia. Diferentes estudos sobre inflamação e fragilidade demonstraram correlação positiva entre aumento de Interleucina 6 (IL-6), TNF e proteína C reativa (PCR) com a fragilidade e com a sarcopenia (Giovannini et al., 2011; Darvin et al., 2014; Hubbard et al., 2010; Gale et al., 2013; 
Lambert et al., 2008). Intervenção física pode ser uma estratégia efetiva para prevenir e tratar a fragilidade, já que a sua prática pode combater até quatro dos cinco critérios propostos por Fried: fraqueza muscular, baixa atividade física, baixa velocidade de caminhada e exaustão (Labra et al., 2015; Clegg et al., 2012). Além disso, estudos demonstram que a prática de exercícios pode reduzir níveis de mediadores da inflamação em idosos frágeis (Lambert et al., 2008; Forti et al., 2014). Porém diferentes revisões sistemáticas (Theou et al., 2015; Frost; Lang; Gelato, 1997; Labra et al., 2015; Cadore et al., 2013; Daniels et al., 2010; Giné-Garriga et al., 2014), apontam que parte dos estudos de intervenção física não apresentam efetividade sobre a fragilidade ou concluem que os benefícios das intervenções limitam-se a atividades funcionais, como sentar e levantar e do equilíbrio e não da fragilidade (Labra et al., 2015; Giné-Garriga et al., 2014). Há uma dificuldade de comparar os estudos de intervenção devido a heterogeneidade do grupo de idosos selecionados, como por exemplo, idosos comunitários e institucionalizados; idosos com diferentes graus de fragilidade, presença de pré-frágeis entre os participantes ou até mesmo estudos com apenas pré frágeis. Outro fator que dificulta a comparação dos estudos de intervenção refere-se aos diferentes métodos existentes de detecção da fragilidade. Esses fatores dificultam a comparação dos estudos e a conclusão quanto à reversão da fragilidade, segundo esses pesquisadores parece que a intervenção física pode reduzir a fragilidade física (Macdonald et al., 2020). De acordo com a literatura há uma falta de consistência nos estudos, possivelmente por não haver uma padronização dos protocolos de intervenção e/ou da definição de fragilidade (Vries et al., 2011), com evidências importantes para uma falta de efetividade da intervenção física sobre a fragilidade (Clegg et al., 2013). O presente estudo pretende analisar se intervenções físicas revertem a fragilidade identificada pelos critérios de Fried em idososcomunitários; e verificar na literatura, o emprego de algum biomarcadorassociado à esta condição, utilizado para avaliação pré e pós-intervenção.

\section{MÉTODOS}

Esta revisão foi conduzida de acordo com os itens de relatório preferencial para revisões sistemáticas e metanálises (declaração prisma) (Salameh et al., 2020) e foi registrada no banco de dados do prospero: CRD42020201516.

Estratégia de Busca: As buscas foram realizadas por dois pesquisadores que de forma independente buscaram artigos nas bases de dados "National Library of Medicine" (PubMed); "Scientific Eletronic Library Online" (Scielo), "Physiotherapy Evidence Database" (PEDro) seguindo os critérios de inclusão e exclusão. Os descritores utilizados no PubMed (MESH terms) e operadores boleanos utilizados com a estratégia de busca descritas no quadro 1 .

Seleção de estudos: Nessa revisão utilizou a estratégia PICO, que permite a partir de uma pergunta delimitar quatro dados como: população $(\mathrm{P})$ - Idosos com idade igual ou acima de 60 anos residentes em comunidades; a intervenção (I) - Intervenção com exercícios fisico em Idosos fragilizados de acordo com os critérios de Fried; a comparação (C) - Realizar a comparação entre os grupos com e sem intervenção fisica analisando se o exercício fisico reverte a sindrome da fragilidade e altera os indice dos biomarcadores analisados; resultado (s) de interesse (O) - É esperado que a intervenção física seja efetiva em idosos gerando mudanças significativas em relaçao sindrome da fragilidade alterando os biomarcadores; design de estudo (S) - Ensaios clinicos Randomizados e/ou controlado (Brown, 2020). A seleção dos artigos foi realizada em duas etapas (títulos e resumos e, posteriormente, texto completo). Para artigos onde houve discordância entre os pesquisadores, um terceiro revisor participou para o desempate. Foram utilizados os seguintes critérios de inclusão: Serem estudos de ensaio clínico randomizado, com participantes com idade igual ou acima de 60 anos; residentes em comunidade; frágeis segundo critérios de Fried; publicado em inglês e/ou português, no periodo de janeiro de 2010 a julho de 2020. Os critérios de exclusão foram: Estudo da fragilidade restrito a um grupo de idosos que apresentam determinada enfermidade, por exemplo, estudo da fragilidade em grupo de idosos diabéticos ou estudo da fragilidade em pacientes cardíacos; Estudo sem o objetivo primário de verificar o efeito da intervenção pelo exercício sobre a fragilidade e/ou seus componentes; estudos duplicados e artigos que não existem versão integral publicada. Os estudos completos foram revisados de forma independente pelos revisores utilizando critérios de avaliação de qualidade dos ensaios clínicos pela escala PEDro (Shiwa et al., 2011) (Tabela 1).

Análise da qualidade dos estudos: Os artigos selecionados foram avaliados, pontuados e qualificados usando a escala PEDro, contendo 11 itens. O item 1 isenta de pontuação, os outros itens obterão uma pontuação de 0 a 10 . O intuito desta escala se fundamenta na avaliação da qualidade metodológica de ensaios clínicos controlados randomizados debatendo com prioridade sua relevância clínica e estatística de maneira compreensível dos resultados para que ocorra com facilidade a reprodução do estudo por outro pesquisado. Os artigos selecionados pelo critério de inclusão através da escala PEDro teriam que possuir pontuação maior ou igual à 5 (Tabela 1) (Shiwa et al., 2011).

Extração e análise dos dados: Os dados relevantes dos estudos selecionados foram extraídos de forma independente por dois avaliadores. O desfecho primário foi a reversão da fragilidade, sendo coletados dados associados aos critérios propostos por Fried (Fried et al., 2001): perda de peso, força de preensão palmar (FPP), velocidade de marcha, exaustão autorelatada, atividade fisica. Dos estudos foram extraídos: (I) os nomes dos autores; (II) ano da publicação, (III) país, tamanho amostral, dados sociodemográficos da população em estudo; (IV) níveis de fragilidade antes e após a intervenção; $(V)$ as formas de intervenção; (VI) resultados da intervenção e (VII) limitações do estudo. Foi também investigada a utilização de biomarcadores (VIII) e sua associação com a reversão do fenótipo. As características e resultados dos estudos incluídos foram sumarizados usando tabelas, complementadas por um resumo narrativo que buscou comparar e avaliar a intervenção utilizada e os principais resultados entre os estudos.

\section{RESULTADOS}

No seguimento de uma ponderação dos artigos derivados dos bancos de dados citados anteriormente, foram encontrados duzentos e quarenta e um artigos de ensaio clínico, sendo sessenta e dois elegíveis para leitura e oito incluídos nesta revisão sistemática (Cameron et al., 2013; Chan et al., 2012; Kim et al., 2015; Li et al., 2010; NG et al., 2015; Tarazona-Santabalbina et al., 2016; NAGAI et al., 2018; Hsieh et al., 2019). A Figura 1 apresenta o fluxograma, com os resultados em cada etapa de busca para identificação dos artigos inclusos e a descrição das causas de exclusão dos artigos.

Qualidade metodológica: Todos os artigos selecionados obtiveram cinco ou mais pontos na escala PeDRO e apresentaram critérios de elegibilidade, com sujeitos alocados aleatoriamente, com quatro trabalhos com alocação oculta (Cameron et al., 2013; CHAN et al., 2012; NG et al., 2015; NAGAI et al., 2018). Nenhum dos estudos revelou diferença significativa entre grupos ao início da intervenção. Em relação à adesão ao tratamento no follow up metade dos estudos (Cameron et al., 2013; Chan et al., 2012; Kim et al., 2015; NG et al., 2015) apresentaram $85 \%$ ou mais de adesão. Todos os estudos adotaram o critério de análise com intenção de tratar de acordo com a Tabela 1. Pode-se observar que todos os estudos possuem baixo risco de viés em relação a alocação aleatória, dados de resultados incompletos, relato de desfecho seletivo ou outras fontes de desfecho; entretanto, alguns dos estudos apresentam-se com risco de viés em relação a ocultação da alocação (Li et al., 2010; $\mathrm{Ng}$ et al., 2015; Nagai et al., 2018), cegamento de pacientes, profissionais (CHAN et al., 2012; Kim et al., 2015) e cegamento dos avaliadores de desfecho (Li et al., 2010; Ng et al., 2015; Tarazona-Santabalbina et al., 2016) (Tabela 1). 
Quadro 1- Estratégia utilizada para busca de estudos de intervenção física em idosos com sindrome da fragilidade

\begin{tabular}{|c|l|}
\hline $\begin{array}{c}\text { BASES DE } \\
\text { DADOS }\end{array}$ & \multicolumn{1}{|c|}{ ESTRATÉGIA DE BUSCA } \\
\hline PEDro & (Older adults) (Physical activity) (Frailtty). \\
\hline Scielo & $\begin{array}{l}\text { "Older adults OR elderly; physical activity; frailty AND year_cluster:("2015" OR } \\
\text { "2018" OR "2013" OR "2019" OR "2020" OR "2011" OR "2016" OR "2017" OR } \\
\text { "2012" OR "2014"). }\end{array}$ \\
\hline PubMed & $\begin{array}{l}\text { ((older adults or elderly AND ((y_10[Filter]) AND (clinicaltrial[Filter]))) AND } \\
\text { (Physical activity AND ((y_10[Filter]) AND (clinicaltrial[Filter]))) AND (frailty } \\
\text { AND ((y_10[Filter]) AND (clinicaltrial[Filter]))). }\end{array}$ \\
\hline
\end{tabular}

Tabela 1. Classificação do risco de viés baseada na escala de PEDro para os estudos incluídos na revisão sistemática. Brasília, DF, 2020

\begin{tabular}{|c|c|c|c|c|c|c|c|c|c|c|c|c|}
\hline \multicolumn{13}{|c|}{ Itens da escala PEDro ${ }^{a}$} \\
\hline ESTUDO & 1 & 2 & & 3 & 4 & 5 & 6 & 7 & 8 & 10 & 11 & PONTUAÇẢO \\
\hline $\begin{array}{c}\text { Cameron et al, } \\
2013\end{array}$ & $\mathrm{~S}$ & $\mathrm{~S}$ & $\mathrm{~S}$ & $\mathrm{~S}$ & $\mathrm{~N}$ & $\mathrm{~N}$ & $\mathrm{~S}$ & $\mathrm{~S}$ & $\mathrm{~S}$ & $\mathrm{~S}$ & $\mathrm{~S}$ & $8 / 10$ \\
\hline $\begin{array}{c}\text { Chan et al, } \\
2012\end{array}$ & $\mathrm{~S}$ & $\mathrm{~S}$ & $\mathrm{~S}$ & S & $\mathrm{N}$ & $\mathrm{N}$ & S & $\mathrm{S}$ & $\mathrm{S}$ & $\mathrm{S}$ & $\mathrm{S}$ & $8 / 10$ \\
\hline $\begin{array}{l}\text { Kim et al, } \\
2015\end{array}$ & $\mathrm{~S}$ & $\mathrm{~S}$ & $\mathrm{~N}$ & S & $\mathrm{N}$ & $\mathrm{N}$ & $\mathrm{S}$ & $\mathrm{S}$ & $\mathrm{S}$ & $\mathrm{S}$ & $\mathrm{S}$ & $7 / 10$ \\
\hline $\begin{array}{l}\text { Li et al, } \\
2010\end{array}$ & $\mathrm{~S}$ & $\mathrm{~S}$ & $\mathrm{~N}$ & $S$ & $\mathrm{~N}$ & $\mathrm{~N}$ & $\mathrm{~N}$ & $\mathrm{~N}$ & $\mathrm{~S}$ & $\mathrm{~S}$ & $\mathrm{~S}$ & $5 / 10$ \\
\hline $\begin{array}{c}\mathrm{Ng} \text { et al, } \\
2015\end{array}$ & $\mathrm{~S}$ & $\mathrm{~S}$ & $\mathrm{~S}$ & S & $\mathrm{N}$ & $\mathrm{N}$ & $\mathrm{S}$ & $\mathrm{S}$ & S & $\mathrm{S}$ & $\mathrm{S}$ & $8 / 10$ \\
\hline $\begin{array}{c}\text { Tarazona- } \\
\text { Santalbalbina et al } \\
2016\end{array}$ & $\mathrm{~S}$ & $\mathrm{~S}$ & $\mathrm{~N}$ & S & $\mathrm{N}$ & $\mathrm{N}$ & $\mathrm{S}$ & $\mathrm{N}$ & $\mathrm{S}$ & $\mathrm{S}$ & $\mathrm{S}$ & $6 / 10$ \\
\hline $\begin{array}{c}\text { Nagai et al, } \\
2018\end{array}$ & $\mathrm{~S}$ & $\mathrm{~S}$ & $\mathrm{~N}$ & $\mathrm{~S}$ & $\mathrm{~N}$ & $\mathrm{~N}$ & $\mathrm{~N}$ & $\mathrm{~N}$ & $\mathrm{~S}$ & $\mathrm{~S}$ & $\mathrm{~S}$ & $5 / 10$ \\
\hline Hsieh et al, 2019 & $\mathrm{~S}$ & $\mathrm{~S}$ & $\mathrm{~N}$ & $\mathrm{~S}$ & $\mathrm{~N}$ & $\mathrm{~N}$ & S & $\mathrm{N}$ & $\mathrm{S}$ & $\mathrm{S}$ & $\mathrm{S}$ & $6 / 10$ \\
\hline
\end{tabular}

Legenda: $\mathrm{S}=$ classificação positiva; $\mathrm{N}=$ classificação negativa; 0 = não há informação disponível; $\mathrm{NA}=$ não se aplica. $\mathrm{T}=$ Total de pontos na escala PEDro 1. Critério de eligibilidade; 2. Alocação randômica; 3. Alocação oculta; 4. Similaridade dos grupos no início; 5. Sujeitos blindados; 6. Terapeutas blindados; 7. Assistentes blindados; 8. Adequado follow up (adesão $\geq 85 \%$ ); 9 . Análise com Intenção de tratar; 10 . Comparação de grupos 11. Medidas de precisão e Variabilidade. * Média dos escores PEDro.

Características dos Participantes e dos estudos: $O$ total de participantes dos estudos selecionados foi de 1505 idosos, com idade média de $77,7 \pm 5,2$ anos de idade, sendo $58,4 \%$ mulheres. metade dos estudos foi realizado com idosos octogenários (Cameron et al., 2013; Kim et al., 2015; Tarazona-Santabalbina et al., 2016; Nagai et al., 2018). a média de participantes por estudo foi de $188 \pm 104,1$ idosos, sendo que apenas um estudo foi realizado com um grupo menor que 100 pessoas (Nagai et al., 2018). a taxa de abandono foi baixa, sendo que apenas dois estudos (nagai et al., 2018; hsieh et al., 2019) apresentaram taxa maior que $20 \%$ (Tabela 1 ). em relação ao tamanho da amostra de idosos frágeis participantes nos estudos inclusos, observou-se que três foram desenvolvidos apenas com idosos frágeis (Cameron et al., 2013; Kim et al., 2015; TarazonaSantabalbina et al., 2016), e os demais com pré frágeis e frágeis (Chan et al., 2012; li et al., 2010; ng et al., 2015; nagai et al., 2018; hsieh et al., 2019). em dois estudos o quantitativo de frágeis foi menor que 15\% dos idosos participantes (Chan et al., 2012; Hsieh et al., 2019).
Em relação ao protocolo de intervenção, grande parte dos estudos propôs uma intervenção física com duração de 12 semanas (Cameron et al., 2013; Chan, d. c. d. et al., 2012; Kim et al., 2015; $\mathrm{Ng}$ et al., 2015; Hsieh et al., 2019), houve estudos que desenvolveram uma intervenção por 6 meses (tarazona-santabalbina et al., 2016; nagai et $a l ., 2018$ ). quanto à adesão às sessões de intervenção (frequência), dois estudos tiveram alto nível de adesão (maior que 85\%) (ng et al., 2015; nagai et al., 2018) grande maioria dos estudos tiveram adesão menor que 60\% (Cameron et al., 2013; Chan et al., 2012; Li et al., 2010; Tarazona-Santabalbina et al., 2016). o estudo de kim et al., 2015 e o de Hsieh et al., 2019 não relatam a taxa de adesão.

Características da intervenção: As características das intervenções desenvolvidas nos estudos estão expressas na Tabela 2. Características dos estudos em relação ao perfil da amostra, intervenção utilizada, reversão da fragilidade e uso de biomarcadores. Ao todo, três estudos promoveram uma intervenção interdisciplinar, envolvendo treinamento físico, apoio nutricional e psicológico (Cameron et al., 2013; Chan et al., 2012; Ng et al., 2015). dois estudos avaliaram o treinamento físico e a intervenção nutricional 
Tabela 2. Características dos estudos em relação ao perfil da amostra, intervenção utilizada, reversão da fragilidade e uso de biomarcadores. Brasília, DF, 2020.

\begin{tabular}{|c|c|c|c|c|c|c|c|c|c|c|}
\hline $\begin{array}{l}\text { Autor/ ano/ } \\
\text { país }\end{array}$ & $\begin{array}{l}\text { N-Início/ } \\
\text { Fim \% de } \\
\text { desistência }\end{array}$ & $\begin{array}{l}\text { Idade } \\
\text { média } \\
\text { (SD) }\end{array}$ & $\begin{array}{l}\text { Participantes do } \\
\text { estudo }\end{array}$ & $\begin{array}{l}\text { Perfil de fragilidade } \\
\text { inicial }\end{array}$ & $\begin{array}{l}\text { Grupo de } \\
\text { Intervenção }\end{array}$ & $\begin{array}{l}\text { Grupo } \\
\text { Controle }\end{array}$ & $\begin{array}{lll}\text { Duração/ } & \text { Frequência } & \text { do } \\
\text { tratamento } & & \end{array}$ & \multicolumn{2}{|c|}{$\begin{array}{l}\text { Reversão da fragilidade/ } \\
\text { \% redução da fragilidade }\end{array}$} & Biomarcador \\
\hline $\begin{array}{ll}\text { CAMERON et } \\
\text { al., } 2013 \\
\text { Austrália }\end{array}$ & \begin{tabular}{|l|}
$241 / 216$ \\
$10,4 \%$
\end{tabular} & $83,3 \pm 5,9$ & $\begin{array}{l}\geq 70 \text { anos } \\
68 \% \text { mulheres }\end{array}$ & $100 \%$ frágeis & $\begin{array}{l}\text { GI }=120 \text { (início) } \rightarrow 108 \text { idosos (término) } \\
\text { Intervenção interdisci plinar Intervenção } \\
\text { individualizada baseado na avaliação } \\
\text { compreensiva geriátrica (CGA) E: TR + TE } \\
+ \text { TF Exercícios domiciliares, por } 9 \text { meses, } \\
\text { após a intervenção }\end{array}$ & $\begin{array}{|ll|}\mathrm{GC}= & 121 \\
\text { (início) } \rightarrow & 117 \\
\text { idosos } & \\
\text { (término) } & \\
& \\
\text { Cuidados } & \\
\text { usuais } & \\
\end{array}$ & $\begin{array}{l}3 \text { meses } \\
2 \mathrm{x} / \mathrm{sem} \\
\text { Follow up: } 9 \text { meses com } \\
\text { exercícios domiciliares }\end{array}$ & \multicolumn{2}{|c|}{$\begin{array}{l}\text { Sim, apenas após } 12 \text { meses: } \\
\text { Redução de: } \\
\text { GI - } 38 \% \\
\text { GC }-24 \%\end{array}$} & ND \\
\hline $\begin{array}{l}\text { CHAN et al., } \\
2012 \\
\text { Taiwan/ China }\end{array}$ & $\begin{array}{l}117 / 101 \\
13,7 \%\end{array}$ & $71,4 \pm 3,7$ & $\begin{array}{l}\geq 65 \text { anos } \\
\text { Sedentários } \\
\text { Mulheres 59\% }\end{array}$ & $\begin{array}{l}13 \% \text { frágeis }(\mathrm{n}=15) \\
87 \% \text { pré frágeis }(\mathrm{n}=102)\end{array}$ & $\begin{array}{l}\text { GI }=84 \text { Intervenção interdisciplinar com } \\
\text { orientações educativas - E: TR, TE e TP } \\
\text { - Suplemento nutricional (N) } \\
\text { - TSP } 3 \text { Grupos: } \\
\text { - TC: } \mathrm{E}+\mathrm{N}+\mathrm{TSP}=28 \text { idosos } \\
\text { - E+ N }=5527 \text { idosos (término) } \\
\text { - TSP }=29 \text { idosos (término) } \\
\end{array}$ & \begin{tabular}{|l|}
$\mathrm{GC}=33$ \\
Apenas \\
orientações \\
educativas
\end{tabular} & $\begin{array}{l}3 \text { meses } \\
3 \mathrm{x} / \mathrm{sem} \\
\text { Sessão: } 60 \mathrm{~min} \\
\text { Follow up: } 9 \text { meses }\end{array}$ & \multicolumn{2}{|c|}{$\begin{array}{l}\text { Sim } \\
\text { Após } 3 \text { meses: } \\
\text { Redução: } \\
\text { GI - TC }-45,0 \% \\
\text { GC }-27,0 \%\end{array}$} & Vit D \\
\hline $\begin{array}{l}\text { KIM et al., } 2015 \\
\text { Japão }\end{array}$ & $\begin{array}{l}131 / 126 \\
3,9 \%\end{array}$ & $80,7 \pm 2,8$ & $\begin{array}{l}\geq 75 \text { anos } \\
\text { Mulheres } 100 \%\end{array}$ & $100 \%$ frágeis & $\begin{array}{l}\text { Intervenção física e/ou nutricional (N) } \\
E: T R+T E+T F l+T M \\
E+N=33 \text { idosos } \\
E=33 \text { idosos } \\
N=32 \text { idosos }\end{array}$ & \begin{tabular}{|l|}
$\mathrm{GC}=33$ \\
Recebeu \\
apenas placebo \\
Sem exercícios \\
\end{tabular} & $\begin{array}{l}\text { meses } \\
\text { 2x/semana } \\
\text { Sessão: } 60 \text { min } \\
\text { Duração: } 3 \text { meses } \\
\text { Follow up }+4 \text { meses }\end{array}$ & \multicolumn{2}{|l|}{$\begin{array}{l}\text { Sim } \\
\text { Após } 3 \text { meses } \\
\text { Reversão de: } \\
\text { E: } 51,5 \% \\
\text { TC: } 57,6 \% \\
\text { GC: } 30,3 \%\end{array}$} & $\begin{array}{l}\text { BDNF } \\
\text { IGF-1 } \\
\text { IGFBP } \\
\text { Miostatina }\end{array}$ \\
\hline $\begin{array}{l}\text { LI et al., } \\
\text { 2010Taiwan/ } \\
\text { China }\end{array}$ & $\begin{array}{l}310 / 265 \\
15 \%\end{array}$ & $78,8 \pm 8.4$ & $\begin{array}{l}\geq 65 \text { anos } \\
\text { Mulheres } 47,7 \%\end{array}$ & $\begin{array}{l}18,3 \% \\
\text { frágeis } \\
81,7 \% \\
\text { pré frágeis }\end{array}$ & $\begin{array}{l}\text { GI }-152 \text { (início) } \rightarrow 127 \text { (término) } \\
17,1 \% \text { frágeis } \\
\text { Intervenção individualizada de acordo com } \\
\text { CGA } \\
\text { Intervenção fisica - ND }\end{array}$ & \begin{tabular}{|l|}
$\mathrm{GC}-158$ \\
(início) $\rightarrow 138$ \\
$($ fim) \\
$19,6 \%$ frágeis \\
$\mathrm{ND}$
\end{tabular} & $\begin{array}{l}\text { ND } \\
\text { Follow up- } 6 \text { meses }\end{array}$ & \multicolumn{2}{|c|}{$\begin{array}{l}\text { Não } \\
\text { Após o follow up } \\
\text { GI }-17,8 \% \text { frágeis } \\
\uparrow 0,7 \% \text { de fragilidade } \\
\text { GC }-24,3 \% \\
\uparrow 4,7 \% \text { de fragilidade }\end{array}$} & ND \\
\hline $\begin{array}{l}\text { NG et al., } 2015 \\
\text { Cingapura }\end{array}$ & $\begin{array}{l}246 / 228 \\
5,4 \%\end{array}$ & $70,0 \pm 4.7$ & $\begin{array}{l}\geq 70 \text { anos } \\
\text { Sedentários } \\
\text { Mulheres } 61,4 \%\end{array}$ & $\begin{array}{l}27,2 \% \text { frágeis } \\
72,8 \% \text { pré frágeis }\end{array}$ & $\begin{array}{l}\text { GI }-196 \\
\text { Intervenção interdisciplinar } \\
\text { Intervenção física: TR + TE. }=48 \\
\text { TCog: }- \text { Treinamento cognitivo }=50 \\
\text { N = Suplemento Nutricional }=49 \\
\text { TC }=\mathrm{E}+\text { TCog }+ \text { N (combinado })=49\end{array}$ & $\begin{array}{l}\text { GC -50 } \\
\text { Cuidados } \\
\text { usuais }\end{array}$ & \begin{tabular}{l|}
3 meses \\
$2 x /$ sem \\
Sessão: $90 \mathrm{~min}$ \\
Duração: $12 \mathrm{sem}$ \\
+12 semanas de exercícios \\
domiciliares \\
Follow up de 9 meses
\end{tabular} & \multicolumn{2}{|c|}{$\begin{array}{l}\text { Sim } \\
\text { Após } 12 \text { meses: } \\
\text { Redução de: } \\
\text { E - } 41,3 \% \\
\text { TC }=47,8 \% \\
\mathrm{GC}=15,2 \%\end{array}$} & ND \\
\hline $\begin{array}{l}\text { TARAZONA- } \\
\text { SANTABALBI } \\
\text { NA et al., } 2016 \\
\text { Espanha }\end{array}$ & $\begin{array}{l}100 / 82 \\
18 \%\end{array}$ & $80,0 \pm 3,6$ & $\begin{array}{l}\geq 70 \text { anos } \\
\text { Sedentários } \\
\text { Idosos do campo } \\
\text { Mulheres } 54 \%\end{array}$ & $100 \%$ frágeis & $\begin{array}{l}\text { GI }-51 \\
\text { Exercícios: } \mathrm{TR}+\mathrm{TE}+\mathrm{TF}+\mathrm{TFl} \\
\text { Todos receberam orientações nutricionais } \\
\text { Suplemento de calciferol para idosos com } \\
\text { calcidiol plasmático }<30 \mathrm{ng} / \mathrm{mL}\end{array}$ & \begin{tabular}{|l|}
$\mathrm{GC}-49$ \\
Cuidados \\
usuais
\end{tabular} & $\begin{array}{l}6 \text { meses } \\
5 \mathrm{x} / \mathrm{sem} \\
65 \mathrm{~min} / \mathrm{sessão} \\
\text { Duração: } 6 \text { meses } \\
\text { Follow up }-12 \text { meses }\end{array}$ & $\begin{array}{l}\text { Sim } \\
\text { Após } 6 \text { meses } \\
\text { GI - } 31,4 \% \\
\text { GC - } 0 \%\end{array}$ & \multicolumn{2}{|c|}{$\begin{array}{l}\text { Calcidiol } \\
\text { Colesterol } \\
\text { Creatinina } \\
\text { D- Dimero, } \\
\text { Fibrinogênio } \\
\text { Fosfatase alcalina } \\
\text { Glicose } \\
\text { Hemoglobina } \\
\text { Proteína Total } \\
\text { Transaminase } \\
\text { TSH } \\
\text { BDNF }\end{array}$} \\
\hline
\end{tabular}




\begin{tabular}{|c|c|c|c|c|c|c|c|c|c|}
\hline $\begin{array}{lll}\text { NAGAI } & \text { et } & \text { al., } \\
2018 & & \\
\text { Japão } & & \end{array}$ & $\begin{array}{l}41 / 32 \\
22 \%\end{array}$ & $81,5 \pm 7,2$ & $\begin{array}{l}\geq 65 \text { anos } \\
\text { Mulheres } 90,5 \%\end{array}$ & $\begin{array}{l}58,5 \% \text { frágeis e } 41,5 \% \\
\text { pré frágeis }\end{array}$ & $\begin{array}{l}\text { GI }-21 \text { (início) } \rightarrow 18 \text { (término) } \\
57 \% \text { frágeis } \\
\text { TR com estímulo para atividade física (AF) } \\
2 \mathrm{x} / \mathrm{sem}\end{array}$ & \begin{tabular}{|lrr}
$\mathrm{GC}$ & - & 20 \\
(início) & $\rightarrow$ & 14 \\
(término) & \\
$60 \%$ frágeis \\
TR r sem \\
estímulo para \\
AF & \\
\end{tabular} & $\begin{array}{l}6 \text { meses } \\
\text { TR } 2 x / \text { sem }\end{array}$ & $\mathrm{NÃO}$ *a $^{\mathrm{a}}$ & ND \\
\hline $\begin{array}{lll}\text { HSIEH } & \text { et al., } \\
2019 & & \\
\text { Taiwan } & & \end{array}$ & $\begin{array}{l}319 / 254 \\
20,4 \%\end{array}$ & $71,6 \pm 5,7$ & $\begin{array}{l}\geq 65 \text { anos } \\
\text { Mulheres } 39,8 \%\end{array}$ & $\begin{array}{l}10,4 \% \text { frágeis } \\
89,6 \% \text { pré frágeis }\end{array}$ & $\begin{array}{l}\text { GI - 3 grupos } \\
\text { E - Exercícios domiciliares (80) - } 13.9 \% \\
\text { frágeis } \\
\mathrm{N} \text { - Nutrição }-83-13,3 \% \text { frágeis } \\
\text { Combinação }(\mathrm{E}+\mathrm{N})-77-6,5 \% \text { frágeis } \\
\text { Exercícios domiciliares que envolvem } \\
\text { alongamento, TF }+\mathrm{TE}+\mathrm{TR} \\
3 \text { a } 7 \text { sessões por semana } \\
\text { Sessão - varia de } 5 \text { a } 60 \text { min }\end{array}$ & $\begin{array}{l}\mathrm{GC}-80 \\
7,5 \% \text { frágeis } \\
\end{array}$ & $\begin{array}{l}3 \text { meses de intervenção } \\
\text { individualizada } \\
\text { Follow up de } 3 \text { meses }\end{array}$ & 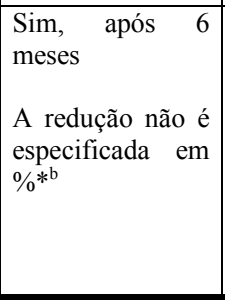 & ND \\
\hline \multicolumn{10}{|c|}{ 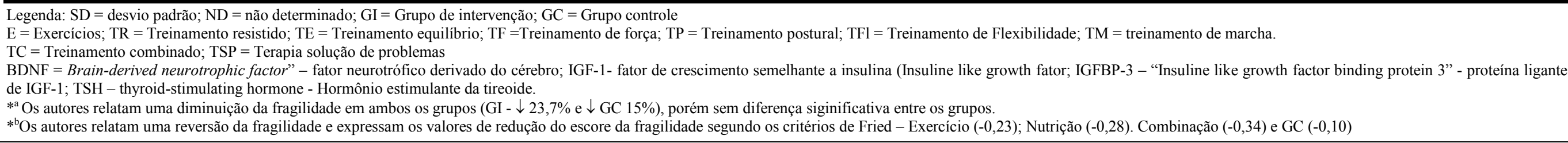 } \\
\hline
\end{tabular}

Tabela 3. Comparação dos efeitos das terapias combinadas nos componentes da fragilidade (critérios de Fried). Brasília, DF, 2020.

\begin{tabular}{|c|c|c|c|c|c|}
\hline Referências & Aumento do peso & $\begin{array}{c}\text { Aumento da Força } \\
\text { muscular }\end{array}$ & $\begin{array}{c}\text { Aumento na } \\
\text { Velocidade de } \\
\text { marcha }\end{array}$ & $\begin{array}{c}\text { Diminuição da } \\
\text { Exaustão auto } \\
\text { relatada }\end{array}$ & $\begin{array}{l}\text { Aumento da } \\
\text { atividade física }\end{array}$ \\
\hline Cameron et al., 2013 & Não & Sim & Sim & Não & Sim \\
\hline Chan et al., 2012 & Não & Não & Sim & Não & Não \\
\hline Kim et al., 2015 & Sim & Não & Sim & Sim & Sim \\
\hline $\mathrm{Ng}$ et al., 2015 & Sim & Sim & Sim & Não & Sim \\
\hline $\begin{array}{c}\text { Tarazona- } \\
\text { Santalbalbina et al., } \\
2016\end{array}$ & Sim & Não & Não & Não & Sim \\
\hline Hsieh et al., 2019 & NR & Sim & Sim & NR & NR \\
\hline
\end{tabular}

Legenda: NR -não relatada. 


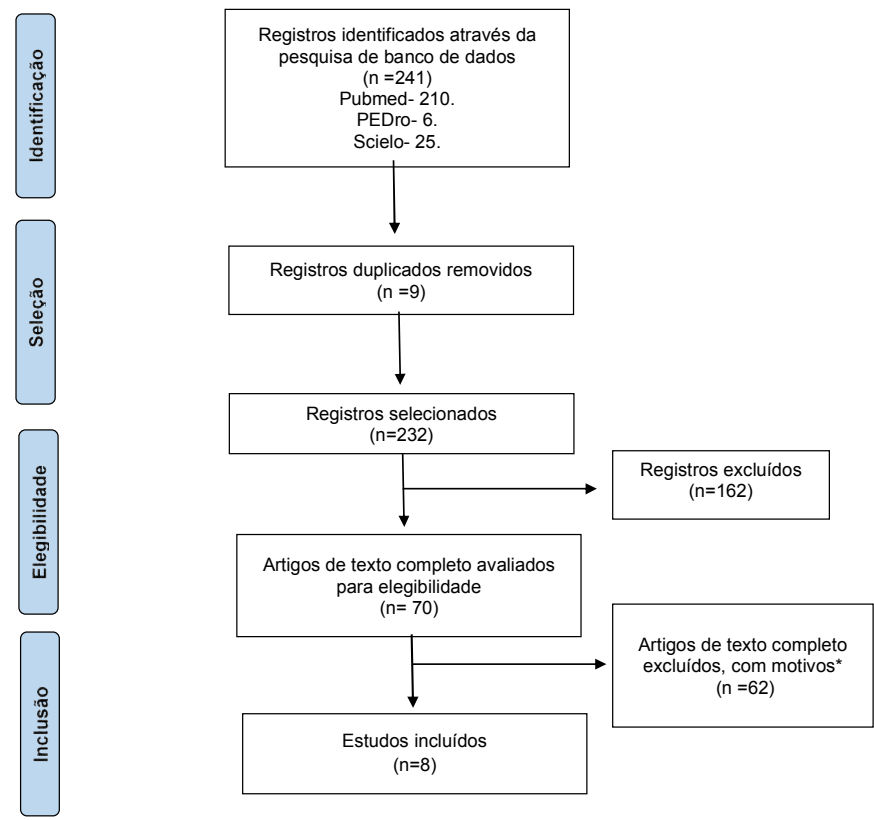

Figura 1. Diagrama de fluxo do Prisma mostrando identificação, triagem, avaliação de elegibilidade e inclusão de estudos

(Kim et al., 2015; Hsieh et al., 2019), enquanto o estudo de tarazonasantabalbina et al., 2016, avaliou somente o treinamento físico, porém foi implementado suporte nutricional para os idosos que necessitavam. os estudos Cameron et al., 2013, $\mathrm{Ng}$, et al., 2015associaram intervenção supervisionada com exercícios domiciliares. Já o estudo de HSIEH et al., 2019, propôs apenas os exercícios domiciliares por três meses, e um follow up por mais três meses, e observaram diferença significativa entre os dois grupos com seis meses. Quanto ao tipo de intervenção física, em regra os estudos desenvolveram treinamento resistido (TR) e de equilíbrio (TE), com exceção ao estudo de Li et al., 2010, que propôs uma intervenção individualizada de acordo com a avaliação geriátrica realizada. Porém, este estudo não descreve o tipo de intervenção física ou complementar que foi realizada, nem a frequência e duração da mesma. Três estudos também executaram treinamento de força (Cameron et al., 2013; Kim et al., 2015; Tarazona-Santabalbina et al., 2016).

Reversão da Fragilidade: Estudos atuais trazem dados relevantes em relação a reversão da fragilidade por meio de intervenção fisica. De acordo com a descrição da Tabela 2, dos oito estudos apenas dois ( $\mathrm{Li}$ et al., 2010; Nagai et al., 2018) não produziram reversão da fragilidade quando comparados com o grupo controle. Os estudos (Cameron et al., 2013; Chan et al., 2012; Kim et al., 2015; NG et al., 2015) envolveram terapia combinada e foram capazes de reverter a fragilidade em $45 \%, 36,3 \%, 47,5 \%, 57,6 \%$ respectivamente, dos idosos participantes. A maioria dos estudos (Cameron et al., 2013; Chan et al., 2012; Kim et al., 2015; Ng, et al., 2015; Hsieh et al., 2019) desenvolveu uma intervenção por doze semanas, com uma frequência de duas vezes por semana. $\mathrm{O}$ estudo que obteve melhor resultado, realizou intervenção física com terapia nutricional específica para cada participante (Kim et al., 2015) (Tabela 2). O estudo que envolve terapia combinada, usando exercícios domiciliares, também relata reversão da fragilidade (HSIEH et al., 2019). Os autores retratam essa reversão significativa através de redução do escore de fragilidade nos grupos, sem apresentar a porcentagem de melhora. Isso dificulta a comparação desse estudo com os demais (Tabela 2). Já no estudode Tarazona-Santabalbina et al., 2016, a intervenção física consistiu em exercícios resistidos, exercícios de equilíbrio, treino de força e flexibilidade, ocorrendo cinco dias da semana, com cada sessão apresentando 65 minutos de duração, por 24 semanas revertendo à fragilidade no grupo de intervenção em $31,4 \%$. Foi à intervenção mais longa de todos os estudos.
Efeitos da intervenção nos componentes da fragilidade: Quanto aos critérios de fragilidade propostos por Fried, seis estudos relataram diferenças entre os grupos de intervenção e controle (Cameron et al., 2013; Chan et al., 2012; Kim et al., 2015; Ng et al., 2015; TarazonaSantabalbina et al., 2016; Hsieh et al., 2019). Todos esses ensaios clinicos propuseram intervenção combinada (intervenção física mais nutricional). O impacto dessa intervenção nos componentes da fragilidade pode ser observado na Tabela 3 Efeitos da intervenção nos componentes da fragilidade (critérios de Fried). Cinco dos seis estudos foram efetivos em melhorar a velocidade de marcha e quatro modificaram a atividade física dos idosos. Tanto o estudo de Kim et al., 2015, quanto de $\mathrm{Ng}$ et al., 2015, melhoraram quatro dos cinco componentes da fragilidade. Já a intervenção realizada no estudo de Tarazona-Santabalbina et al., 2016, melhorou dois critérios: aumento de peso e de atividade física. O estudo de Hsieh et al., 2019, não apresenta resultados de todos os critérios da fragilidade, mas relata uma melhora na força de preensão palmar e na velocidade de marcha.

Biomarcadores: Três estudos avaliaram algum biomarcador (Chan et al., 2012; Kim et al., 2015; Tarazona-Santabalbina et al., 2016) em seus estudos de intervenção (tabela 2). o estudo de chan et al., 2012, dosou apenas a vitamina d, já o estudo de Kim et al., 2015, dosou no soro: o fator de crescimento do sistema nervoso "brain-derived neurotrophic factor" (bdnf); fator de crescimento semelhante a insulina (insuline like growth factor -igf-1), sua proteína transportadora (insuline like growth factor binding protein 3 - igfbp3) e miostatina. já o estudo de Tarazona-Santabalbina et al., 2016avaliou vários marcadores bioquímicos, inclusive bdnf. $\mathrm{O}$ estudo de Chan et al., 2012 verificou um aumento sorológico significativo de $25(\mathrm{OH})$ vitamina $\mathrm{D}$ no grupo de intervenção do que no grupo controle $(4,9 \pm 7,7$ versus $1,2 \pm 5,4 ; \mathrm{p}=0,006)$. Tanto no estudo de Kim et al., 2015 quanto de Tarazona-Santabalbina et al., 2016 houve um aumento de BDNF, porém tarazona-santabalbina et al., 2016 não observou diferença significativa desse aumento entre o grupo de intervenção e o grupo controle. Já o estudo de Kim et al., 2015 verificou diferença significativa entre os grupos $(p=0,013)$. Nenhum dos marcadores avaliados por Tarazona-Santabalbina et al., 2016 apresentou alguma diferença significativa. Já no estudo de Kim et al., 2015 além do BDNF, houve diferença significativa para a miostatina e para a razão IGF-1/IGFBP-3, que apresentaram menores valores. O BDNF aumenta a plasticidade cerebral, através da promoção da neurogênese, da proliferação celular, da geração de sinapses no hipocampo (Yoon; Lee; Song, 2018). Embora a inflamação participe do processo de fragilidade, nenhum dos estudos analisados avaliou algum marcador de inflamação, como proteína $\mathrm{C}$ reativa $(\mathrm{PCR})$ ou citocinas.

\section{DISCUSSÃO}

A análise dos artigos permite afirmar que a intervenção física e a intervenção combinada (física associada a nutrição ou psicológica) são capazes de reverter a fragilidade, demonstrando ser essa síndrome reversível. O principal critério modificado através das intervenções combinadas propostas é o aumento da velocidade de marcha, seguida do aumento da atividade física. Isso resulta da intervenção fisica e da intervenção nutricional que melhora o desempenho muscular. comparando os estudos observa que duas intervenções propostas (Kim et al., 2015; $\mathrm{Ng}$ et al., 2015), ambos com populações asiáticas, foram as mais efetivas em modificar os critérios de fragilidade e reduzir a fragilidade, sendo que o estudo de Kim et al., 2015 foi que alcançou uma maior diminuição nos scores que pontuam a fragilidade no idoso com uma intervenção em três meses sendo o único que mostrou uma diminuição da exaustão (fadiga) auto relatada. os estudos de Kim et al., 2015 e de $\mathrm{Ng}$ et al., 2015 propuseram exercícios com treinamento resistido, treinamento equilíbrio e treinamento de força, corroborando com os estudos de yoon; lee; song, 2018 e de Cadore et al., 2013, porém o estudo de yoon; lee; song, 2018 não usou os critérios de fried para avaliar a fragilidade e o de Cadore et al., 2013 avaliou idosos institucionalizados. o estudo de nagai et al., 2018 também adotou treinamento resistido em ambos os grupos (controle e de intervenção) com a diferença do folheto 
educativo e o material impresso com os acelerômetros por meio do sistema de feedback para aumento do desempenho na intervenção fisica. Observou-se uma redução da fragilidade em ambos os grupos, porém sem diferença estatistica significativa entre os mesmos. Isso demonstra que a redução ocorreu devido a intervenção física por meio do treinamento resistido e não pelo aumento da prática da atividade proposta. Apenas um dos estudos selecionados propôs intervenção com exercícios domiciliares, sem usar outro tipo de intervenção física (Hsieh et al., 2019), obtendo uma reversão dos níveis de fragilidade com seis meses de intervenção. Há na literatura, uma revisão sistemática sobre a intervenção com exercícios domiciliares em idosos frágeis. O desfecho primário analisado nessa revisão foi a melhora na mobilidade e na qualidade de vida, sendo que segundo os autores há uma evidência preliminar de que esse tipo de intervenção melhora a mobilidade em idosos com fragilidade moderada, mas não com fragilidade severa. Não houve avaliação da reversão da fragilidade (Clegg et al., 2014). Exercícios domiciliares segundo uma revisão cochrane de 2005 (Ashworth et al., 2005) tem maior adesão dos idosos que intervenções físicas. Estudos sobre a eficiência desse tipo intervenção no tratamento de idosos frágeis se faz necessário, pois muitos idosos têm dificuldade de locomoção, sendo um programa de exercícios domiciliares mais plausíveis de terem adesão e ter menor custo para a área de saúde pública. Esta revisão sistemática também mostra que há poucos estudos de intervenção com idosos comunitários frágeis, de acordo com critérios de Fried. Isto corrobora os dados observados em outras revisões sistemáticas sobre o tema, onde os autores apontam a grande diversidade de instrumentos para avaliação da fragilidade e que dificultava a comparação dos estudos (Labra et al., 2015; Clegg et al., 2012; Cadore et al., 2013; Giné-Garriga et al., 2014). Outro fato observado é que em apenas três ensaios clinicos foram desenvolvidos com idosos frágeis. Os demais estudos tinham uma grande porcentagem de idosos pré-frágeis. Isso corrobora a dificuldade de se fazer estudos de intervenção com idosos frágeis. Segundo Provencher et al., 2014 uma das dificuldades de estudos de intervenção com idosos frágeis é o recrutamento e a adesão ao tratamento, devido às condições de saúde que dificultam, por exemplo, a locomoção (fadiga, velocidade de marcha diminuído)

Quanto à existência de um biomarcador, não foi detectado nesses estudos, embora a inflamação seja um ponto chave na síndrome da fragilidade. nenhum dos estudos dessa revisão observou o efeito da intervenção na fragilidade e no processo de inflamação. um dos pontos fortes desta revisão é ter incluído apenas artigos de alta qualidade metodológica, ter utilizado como critério de inclusão, apenas estudos que usaram o instrumento de fried para identificação da fragilidade permitindo a comparação de dados. outro ponto forte foi a exclusão de estudos com idosos institucionalizados ou hospitalizados, que geralmente são mais dependentes e com condições clínicas instáveis. diferentes revisões (Labra et al., 2015; Clegg et al., 2012; Provencher et al., 2014), inclusive uma com metanálise, mencionam a dificuldade de comparação devido os estudos adotarem diferentes critérios de identificação da fragilidade e de incluírem idosos institucionalizados ou hospitalizados (Macdonald et al., 2020).

Algumas limitações deste estudo podem ser apontadas: escassez de estudos com idosos fragilizados, avaliados pelos critérios de Fried; o tamanho amostral reduzido devido a dificuldade de adesão dos idosos frágeis; a diversidade das intervenções propostas e de adesão ao tratamento; diferentes graus de fragilidade, de faixa etária e proporção de gênero entre os idosos; estudos desenvolvidos com diferentes populações, sendo a maioria de origem asiática, em diferentes graus de comorbidades entre os idosos dos estudos. A grande heterogeneidade dos estudos dificulta a capacidade de sintetizar o design mais efetivo de um programa de exercícios físicos e os efeitos da intervenção proposta. Pesquisas de intervenção física com um maior número de idosos são necessárias para verificar a efetividade na reversão da fragilidade e modificar outras variáveis associadas a síndrome da fragilidade, que é multifatorial. Além disso, desenvolver pesquisas que identifiquem um biomarcador para monitorar a fragilidade são necessárias para o acompanhamento do tratamento do idoso frágil. Estes estudos poderão fundamentar as futuras políticas públicas de atenção à saúde do idoso.

\section{CONCLUSÕES}

Por meio deste estudo foi possível evidenciar que intervenções combinadas (intervenção física associada a outra modalidade de intervenção) e intervenções físicas possuem um potencial na reversão da fragilidade em idosos, sendo melhor a intervenção combinada, que envolve treinamento resistido. $O$ principal critério de Fried modificado pela intervenção foi o aumento da velocidade de marcha $\mathrm{O}$ critério de exaustão foi o menos modificado. Não foi possível identificar algum biomarcador nos estudos, que possa monitorar a fragilidade.

\section{REFERENCES}

Ashworth, N. L. et. al.. Home versus center based physical activity programs in older adults. Cochrane Database of Systematic Reviews. 2005.

Assumpção, A. M.; DIAS R. C.. Efeitos da fisioterapia na prevenção e na reversão da fragilidade em idosos: revisão sistemática. Geriatrics, Gerontology and Aging, v. 3, n. 3, p. 117-130. 2009.

Brown, D. A.. Review of the PubMed PICO tool: using evidencebased practice in health education. Health promotion practice, 21(4): 496-498, 2020.

Cadore, E. L. et. al..Effects of different exercise interventions on risk of falls, gait ability, and balance in physically frail older adults: a systematic review. Rejuvenation research, v. 16, n. 2, p. 105-114. 2013.

Cameron, I. D. et. al.. A multifactorial interdisciplinary intervention reduces frailty in older people: randomized trial. BMC medicine, v. 11, n. 1, p. $65,2013$.

Chan, D. C. D. et. al. A pilot randomized controlled trial to improve geriatric frailty. BMC geriatrics, v. 12, n. 1, p. 58-70, 2012.

Chen, X.; Mao, G.; NG, S. X. Frailty syndrome: an overview. Clinical interventions in aging, v. 9, p. 433, 2014.

Clegg, A. et al., 2014. The Home-based Older People's Exercise (HOPE) trial: a pilot randomised controlled trial of a homebased exercise intervention for older people with frailty. Age and ageing, v. 43, n. 5, p. 687-695, 2014.

Clegg, A. et. al.Frailty in elderly people. The lancet, v. 381, n. 9868 , p. 752-762, 2013.

Clegg, A. P. et. al. Do home-based exercise interventions improve outcomes for frail older people? Findings from a systematic review. Reviews in clinical gerontology, v. 22, n. 1, p. 68,2012.

Collard, R. M. et. al.. Prevalence of frailty in community $\square$ dwelling older persons: a systematic review. Journal of the American Geriatrics Society, v. 60, n. 8, p. 1487-1492,2012.

Daniels, R. et. al.. Interventions to prevent disability in frail community-dwelling older persons: an overview. European Journal of Ageing, v. 7, n. 1, p. 37-55, 2010.

Darvin, K. et. al.. Plasma protein biomarkers of the geriatric syndrome of frailty. Journals of Gerontology Series A: Biomedical Sciences and Medical Sciences, v. 69, n. 2, p. 182$186,2014$.

Dent, E.; Kowal, P.; Hoogendijk, E. O., 2016.Frailty measurement in research and clinical practice: a review. European journal of internal medicine, v. 31, p. 3-10, 2016.

Forti, L. N. et. al. Strength training reduces circulating interleukin-6 but not brain-derived neurotrophic factor in community-dwelling elderly individuals. Age, v. 36, n. 5, p. 9704, 2014.

Fried, L. P. et. al. Frailty in older adults: Evidence for a phenotype. The Journals of Gerontology Series A: Biological Sciences and Medical Sciences, v. 56, n. 3, p. M146-M157, 2001.

Fried, L. P. et. al. Untangling the concepts of disability, frailty, and comorbidity: implications for improved targeting and care. The Journals of Gerontology Series A: Biological Sciences and Medical Sciences, v. 59, n 3, p. M255-M263, 2004. 
Gale, C. R. et. al. Inflammatory markers and incident frailty in men and women: the English Longitudinal Study of Ageing. Age, v. 35 , n. 6 , p. 2493-2501, 2013

Giné-Garriga, M. et. al.. Physical exercise interventions for improving performance-based measures of physical function in communitydwelling, frail older adults: a systematic review and metaanalysis. Archives of physical medicine and rehabilitation, v. 95, n. 4, p. 753-769, 2014.

Giovannini, S. et. al. Interleukin $\square 6, C \square$ reactive protein, and tumor necrosis factor $\square$ alpha as predictors of mortality in frail, community $\square$ living elderly individuals. Journal of the American Geriatrics Society, v. 59, n. 9, p. 1679-1685, 2011.

Hsieh, T. J. et. al. Individualized home-based exercise and nutrition interventions improve frailty in older adults: a randomized controlled trial. International Journal of Behavioral Nutrition and Physical Activity, v. 16, n. 1, p. 119, 2019.

Hubbard, R. E. et. al.Frailty, body mass index, and abdominal obesity in older people. Journals of Gerontology Series A: Biomedical Sciences and Medical Sciences, v. 65, n.4, p. 377-381, 2010.

$\mathrm{Kim}, \mathrm{H}$. et. al. Effects of exercise and milk fat globule membrane (MFGM) supplementation on body composition, physical function, and hematological parameters in community-dwelling frail Japanese women: a randomized double blind, placebocontrolled, follow-up trial.PLoS One, v. 10, n. 2, p. e0116256, 2015.

Labra, C. et. al.. Effects of physical exercise interventions in frail older adults: a systematic review of randomized controlled trials. BMC geriatrics, v. 15, n.1, p. 154, 2015.

Lambert, C. P. et. al. Exercise but not diet-induced weight loss decreases skeletal muscle inflammatory gene expression in frail obese elderly persons. Journal of Applied Physiology, v. 105, n. 2, p. 473-478, 2008.

$\mathrm{Li}, \mathrm{C} . \mathrm{M}$. et. al.The effectiveness of a comprehensive geriatric assessment intervention program for frailty in communitydwelling older people: a randomized, controlled trial. Archives of Gerontology and geriatrics, v. 50, p. S39-S42, 2010.

Macdonald, S. H. F. et. al.. Primary care interventions to address physical frailty among community-dwelling adults aged 60 years or older: A meta-analysis. PloS one, v. 15, n. 2, p. e0228821, 2020.

Nagai, K. et. al.Physical activity combined with resistance training reduces symptoms of frailty in older adults: A randomized controlled trial. Archives of gerontology and geriatrics, v. 76, p. 41-47, 2018.
$\mathrm{Ng}, \mathrm{T}$. P. et. al.Nutritional, physical, cognitive, and combination interventions and frailty reversal among older adults: a randomized controlled trial. The American journal of medicine, v. 128, n. 11, p. 1225-1236, 2015.

Provencher, V. et. al. Challenges and strategies pertaining to recruitment and retention of frail elderly in research studies: a systematic review. Archives of Gerontology and Geriatrics, v. 59, n. 1, p. 18-24, 2014.

Salameh, J. P. et. al. 2020. Preferred reporting items for systematic review and meta-analysis of diagnostic test accuracy studies (PRISMA-DTA): explanation, elaboration, and checklist. BMJ, v. 370.

Shiwa, S. R. et. al.PEDro: the physiotherapy evidence database. Fisioterapia em Movimento, v. 24, n. 3, p.523-533, 2011.

Tarazona-Santabalbina, F. J. et. Al.A multicomponent exercise intervention that reverses frailty and improves cognition, emotion, and social networking in the community-dwelling frail elderly: a randomized clinical trial. Journal of the American Medical Directors Association, v. 17, n. 5, p. 426-433., 2016.

Theou, O. et. al.. Modifications to the frailty phenotype criteria: Systematic review of the current literature and investigation of 262 frailty phenotypes in the Survey of Health, Ageing, and Retirement in Europe. Ageing research reviews, v. 21, p.78-94, 2015.

Varadhan, R. et. al..Stimulus-response paradigm for characterizing the loss of resilience in homeostatic regulation associated with frailty. Mechanisms of ageing and development, v. 129, n. 11, p. 666-670, 2008.

Vries, N. M. et. al.. Outcome instruments to measure frailty: a systematic review. Ageing research reviews, v.10, n. 1, p. 104$114,2011$.

Wrann, C. D. et. al. Exercise induces hippocampal BDNF through a $P G C-1 \alpha / F N D C 5$ pathway. Cell metabolismo, v. 18, n.5, p. 649659, 2013.

Yoon, D. H.; Lee, J. Y.; Song, W. Effects of resistance exercise training on cognitive function and physical performance in cognitive frailty: a randomized controlled trial. The journal of nutrition, health \& aging, v. 22, n. 8, p. 944-951, ., 2018 